\title{
Evaluation method for spatial straightness errors based on minimum zone condition
}

\author{
Qing Zhang a, K.C. Fan ${ }^{\mathrm{b}, *}$, Zhu $\mathrm{Li}^{\mathrm{a}}$ \\ ${ }^{a}$ School of Mechanical Engineering, Huazhong University of Science and Technology, Wuhan, China \\ ${ }^{\mathrm{b}}$ Department of Mechanical Engineering, National Taiwan University, Taipei, Taiwan
}

Received 28 October 1997; received in revised form 9 June 1998; accepted 16 April 1999

\begin{abstract}
A nonlinear mathematical model for spatial straightness error evaluation based on the minimum zone condition is established in this paper. According to the error analysis, it is proved that the mathematical model for spatial straightness error evaluation cannot be linearized. A criterion for verification of the existence and uniqueness of the minimum zone solution is proposed. A new computational method is also proposed, and practical examples are given. Finally, the correctness of this method is demonstrated using a geometrical solution. This new method is convenient for computation of uniqueness and exactness of the minimum zone solution. (C) 1999 Elsevier Science Inc. All rights reserved.
\end{abstract}

\section{Introduction}

The term "straightness error" generally refers to the deviation between an actual line and an ideal line. In the ISO/1101 [1] standard, two types of straightness errors are specified; namely, planar straightness errors and spatial straightness errors. Evaluations of planar straightness errors have been thorough and mature, as found in many reports [2-7]. At present, however, significant research on spatial straightness errors is scant. Huang [8] proposed the smallest parallelepiped enclosure method for spatial straightness error evaluation from the composition of two orthogonal planar straightness errors (vertical and horizontal). This method provides only a part of the solutions conforming to ISO specifications, because it cannot guarantee that the evaluated error has the same value in every direction. Therefore, the spatial straightness error of a minimum cylindrical enclosure remains to be studied.

In practice, most lines are spatial lines; therefore, the evaluation of straightness errors should be carried out in a threedimensional (3-D) space. Definition of the spatial straightness errors of a minimum cylindrical enclosure as defined in ISO/

$$
1186 .
$$

* Corresponding author. Tel.: +886-2-362-0032; fax: +886-2-364-

E-mail address: fan@ccms.ntu.edu.tw (K.C. Fan)
1101 is: the minimum diameter of a cylinder that can enclose the whole measured data. According to such a definition, the error evaluation must be based on the minimum zone concept so that the spatial straightness errors can be expressed as the diameter of the minimum enclosed cylinder.

In this study, a nonlinear mathematical model for spatial straightness error evaluation is established. It can be proved that this model cannot be linearized. The criterion for the verification of the existence and uniqueness of the minimum zone solution is proposed. A new computational method to attain such a solution is also proposed. Finally, correctness of this method is demonstrated using a graphic representation. This method is comprehensive in its theoretical foundation and is convenient in computation.

\section{Mathematical model}

We assume that the measuring datum line is the $Z$-axis, as shown in Fig. 1. The measured points of the spatial line are expressed by the cylindrical coordinate $(r, \theta, z)$, where $r$ is the actual deviation between the measured point and the measuring datum line, $\theta$ is the phase angle from the $x$-axis, and $z$ is the height of the point location. Because, in practice, the actual measured line will be in misalignment with the measuring datum line, the minimum enclosure cylinder 


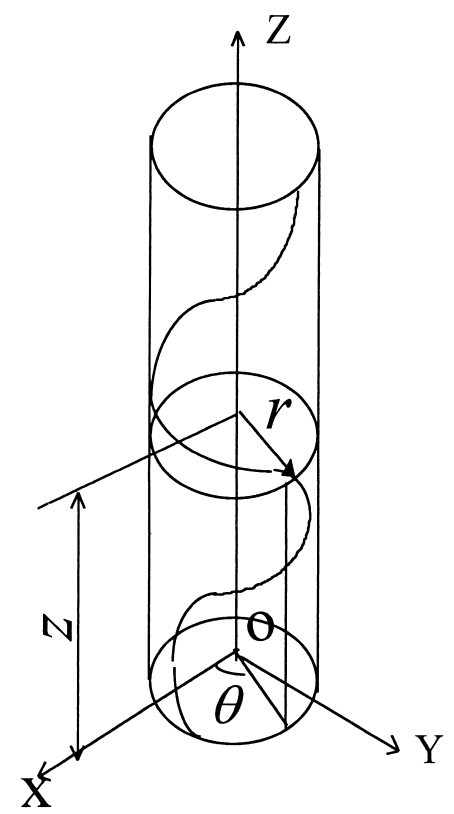

Fig. 1. The coordinate expression of the cylindrical spatial line.

of the spatial straightness errors can be expressed as a titled cylinder, as shown in Fig. 2, with its central axis $o^{\prime} o^{\prime \prime}$.

The $o^{\prime} o^{\prime \prime}$ axis intersects the base plane $(z=0)$ at point $\left(-x_{0}\right.$, $\left.-y_{0}\right)$, as shown in Fig. 3. The angle included between the $o^{\prime} o^{\prime \prime}$ axis and the $Z$-axis on the $X O Z$ plane is $\alpha$, and on the $Y O Z$ plane is $\beta$. The distance between the measured point $P$ and the $o^{\prime} o^{\prime \prime}$ axis at any particular height $z$ is approximate to $R$, which can be expressed by the following form.

$R=$

$$
\sqrt{\left(r \cos \theta+x_{0}+z \tan \alpha\right)^{2}+\left(r \sin \theta+y_{0}+z \tan \beta\right)^{2}}
$$

$R$ is a function of two groups of variables, which are $(r, \theta, z)$ and $\left(x_{0}, y_{0}, \alpha, \beta\right)$. Group $(r, \theta, z)$ indicates the measured point with respect to the datum line and is called the form variables here, expressed by parameter $v$. Group $\left(x_{0}, y_{0}, \alpha, \beta\right)$ describes the location and direction of the central axis $o^{\prime} o^{\prime \prime}$ of the tilted cylinder and is called the alignment variables, expressed by parameter $u$. Because the value of $u$ is very small in practical precision measurement, we assume $\tan \alpha \approx \alpha$, $\tan \beta \approx \beta$. Eq. (1) can then be expressed as follows:

$$
\begin{aligned}
R(v ; u) & =R\left(r, \theta, z ; x_{0}, y_{0}, \alpha, \beta\right) \\
& =\sqrt{\left(r \cos \theta+x_{0}+\alpha z\right)^{2}+\left(r \sin \theta+y_{0}+\beta z\right)^{2}}
\end{aligned}
$$

For a certain $u$ value, the maximal value of $\bar{R}(u)$ represents the maximal diameter of the outer enclosure cylinder relative to the value of $u$, and can be denoted as below:

$$
\begin{aligned}
\bar{R}(u) & =\max \{R(v): v \in V\} \\
& =\max \left\{R_{1}, R_{2}, \ldots, R_{i}, \ldots R_{n}\right\}
\end{aligned}
$$

where $V$ represents the set of form variables of measured points, $i \in\{1,2, \ldots, n\}$, and $n$ represents the number of measured points.

The essence of evaluating the spatial straightness error based on the minimum zone condition is to find the value of $u=\left(x_{0}, y_{0}, \alpha, \beta\right)^{T}$, which makes $\bar{R}(u)$ minimal. This is essentially the following min-max problem to find the location and direction of the smallest outer enclosure cylinder.

$$
\left\{\begin{array}{cc}
\operatorname{minimize} & \bar{R}(u) \\
\text { s.t. } & u \in E^{4}
\end{array}\right.
$$

The spatial straightness error can then be expressed by

$$
f=2 \bar{R}(u)
$$

\section{Error analysis using linearized model}

The straightness errors of a spatial line, as indicated in Eq. (2), are obviously a nonlinear function. The nonlinear model is usually simplified by linearization to solve problems quickly with approximation. The degree of the model error is usually estimated to determine whether a practical nonlinear problem can be linearized. Its influence on the applicability of the linear model should also be considered in any engineering problem. In this study, the linearization of Eq. (2) is analyzed as follows.

With proper alignment of the actual measured line and the measuring datum line, the form parameter $u=\left(x_{0}, y_{0}\right.$, $\alpha, \beta)^{T}$ must be very small in comparison to the straightness error $R$. Function $R(v ; u)$ can be approximated by the Taylor series expansion with $u=0$.

$$
\begin{aligned}
R(v ; u)= & R(v ; 0)+\nabla R_{u}(v ; 0) u \\
& +\frac{1}{2} u^{T} H_{u}(v ; 0) u+\ldots
\end{aligned}
$$

Neglecting the higher-order terms, it can be simplified using the following linear model.

$$
R(v ; u)=R(v ; 0)+\nabla R_{u}(v ; 0) u
$$

where $R(v ; 0)=r$. The first-order partial derivative of $R(v$; $u$ ) at $u=0$ is:

$$
\nabla R_{u}(v ; 0)=(\cos \theta, \sin \theta, z \cos \theta, z \sin \theta)
$$

Therefore,

$R(v ; u)=r+x_{0} \cos \theta+y_{0} \sin \theta+\alpha z \cos \theta+\beta z \sin \theta$

Eq. (7) is the approximated linear model. The model error can be estimated using the second-order term:

$$
e \approx \frac{1}{2} u^{T} H_{u}(v ; 0) u
$$

where, 


$$
H_{u}(v ; 0)=\left[\begin{array}{cccc}
\frac{\partial^{2} R}{\partial x_{0}{ }^{2}} & \frac{\partial^{2} R}{\partial x_{0} \partial y_{0}} & \frac{\partial^{2} R}{\partial x_{0} \partial \alpha} & \frac{\partial^{2} R}{\partial x_{0} \partial \beta} \\
\frac{\partial^{2} R}{\partial x_{0} \partial y_{0}} & \frac{\partial^{2} R}{\partial y_{0}^{2}} & \frac{\partial^{2} R}{\partial y_{0} \partial \alpha} & \frac{\partial^{2} R}{\partial y_{0} \partial \beta} \\
\frac{\partial^{2} R}{\partial x_{0} \partial \alpha} & \frac{\partial^{2} R}{\partial y_{0} \partial \alpha} & \frac{\partial^{2} R}{\partial \alpha^{2}} & \frac{\partial^{2} R}{\partial \alpha \partial \beta} \\
\frac{\partial^{2} R}{\partial x_{0} \partial \beta} & \frac{\partial^{2} R}{\partial y_{0} \partial \beta} & \frac{\partial^{2} R}{\partial \alpha \partial \beta} & \frac{\partial^{2} R}{\partial \beta^{2}}
\end{array}\right] \mid u=0
$$

Each of the second-order partial derivative of $R(v ; u)$ at $u=0$ can be obtained as
Substituting the above data into Eq. (10), we get the maximum value of $e$ as $1.997 \mu \mathrm{m}$ at $\theta=80.4^{\circ}$. Thus, it is obvious that the model error is high, which indicates the simplified linear model is poor. To summarize the above analysis we can conclude that the evaluation of the spatial straightness errors should use the original nonlinear model.

\section{Criterion for minimum zone condition}

From the above analysis, we know that $R(v ; u)$ is a nonlinear function of $u$. Assuming $S \subseteq E^{n}$ (a nonempty open convex set), it can be proved that for every point of

$$
\begin{array}{rlrl}
\left.\frac{\partial^{2} R}{\partial x_{0}^{2}}\right|_{u=0} & =\frac{\sin ^{2} \theta}{r} ; & \left.\frac{\partial^{2} R}{\partial y_{0}^{2}}\right|_{u=0} & =\frac{\cos ^{2} \theta}{r} ; \\
\left.\frac{\partial^{2} R}{\partial \alpha^{2}}\right|_{u=0} & =\frac{z^{2} \sin ^{2} \theta}{r} ; & \left.\frac{\partial^{2} R}{\partial \beta^{2}}\right|_{u=0} & =\frac{z^{2} \cos ^{2} \theta}{r} ; \\
\left.\frac{\partial^{2} R}{\partial x_{0} \partial y_{0}}\right|_{u=0} & =-\frac{\cos \theta \sin \theta}{r} ; & \left.\frac{\partial^{2} R}{\partial x_{0} \partial \alpha}\right|_{u=0} & =\frac{z \sin ^{2} \theta}{r} ; \\
\left.\frac{\partial^{2} R}{\partial x_{0} \partial \beta}\right|_{u=0} & =-\frac{z \cos \theta \sin \theta}{r} ; & \left.\frac{\partial^{2} R}{\partial y_{0} \partial \alpha}\right|_{u=0}=-\frac{z \cos \theta \sin \theta}{r} ; \\
\left.\frac{\partial^{2} R}{\partial y_{0} \partial \beta}\right|_{u=0}=\frac{z \cos ^{2} \theta}{r} ; & \left.\frac{\partial^{2} R}{\partial \alpha \partial \beta}\right|_{u=0}=-\frac{z^{2} \cos \theta \sin \theta}{r}
\end{array}
$$

Substituting Eqs. (9) into Eq. (8), we have:

$$
\begin{aligned}
e & =\frac{1}{2 r}\left[x_{0}, y_{0}, \alpha, \beta\right]\left[\begin{array}{cccc}
\sin ^{2} \theta & -\cos \theta \sin \theta & z \sin ^{2} \theta & -z \cos \theta \sin \theta \\
-\cos \theta \sin \theta & \cos ^{2} \theta & -z \cos \theta \sin \theta & z \cos ^{2} \theta \\
z \sin ^{2} \theta & -z \cos \theta \sin \theta & z^{2} \sin ^{2} \theta & -z^{2} \cos \theta \sin \theta \\
-z \cos \theta \sin \theta & z \cos ^{2} \theta & -z^{2} \cos \theta \sin \theta & z^{2} \cos ^{2} \theta
\end{array}\right]\left[\begin{array}{c}
x_{0} \\
y_{0} \\
\alpha \\
\beta
\end{array}\right] \\
& =\left[\left(x_{0}+\alpha z\right) \sin \theta-\left(y_{0}+\beta z\right) \cos \theta\right]^{2} /(2 r)
\end{aligned}
$$

In this case, the model error can be shown to be considerably high and is deemed not negligible.

With the data given in Example 1 of section 6, we can attain the following results: $u \in S$, the Hessian matrix $H_{u}(v ; u)$ of $R(v ; u)$ at $u$ is semipositive definite in $E^{n}$; that is, all its sequential principal minors are non-negative. This is proved as follows.

$$
\begin{aligned}
& x_{0}=4.6408 \mu \mathrm{m}, \quad y_{0}=4.7438 \mu \mathrm{m}, \quad \alpha=-0.000019 \mathrm{rad} \text {, } \\
& \beta=-0.000006 \text { rad. }, \quad z_{\max }=60 \times 10^{4} \mu \mathrm{m}, \quad f=23.5262 \mu \mathrm{m} \text {. }
\end{aligned}
$$




$$
\begin{aligned}
H_{u}(v ; u) & =\left[\begin{array}{cccc}
\frac{\partial^{2} R}{\partial x_{0}{ }^{2}} & \frac{\partial^{2} R}{\partial x_{0} \partial y_{0}} & \frac{\partial^{2} R}{\partial x_{0} \partial \alpha} & \frac{\partial^{2} R}{\partial x_{0} \partial \beta} \\
\frac{\partial^{2} R}{\partial x_{0} \partial y_{0}} & \frac{\partial^{2} R}{\partial y_{0}{ }^{2}} & \frac{\partial^{2} R}{\partial y_{0} \partial \alpha} & \frac{\partial^{2} R}{\partial y_{0} \partial \beta} \\
\frac{\partial^{2} R}{\partial x_{0} \partial \alpha} & \frac{\partial^{2} R}{\partial y_{0} \partial \alpha} & \frac{\partial^{2} R}{\partial \alpha^{2}} & \frac{\partial^{2} R}{\partial \alpha \partial \beta} \\
\frac{\partial^{2} R}{\partial x_{0} \partial \beta} & \frac{\partial^{2} R}{\partial y_{0} \partial \beta} & \frac{\partial^{2} R}{\partial \alpha \partial \beta} & \frac{\partial^{2} R}{\partial \beta^{2}}
\end{array}\right] \\
= & \frac{1}{R^{3}}\left[\begin{array}{cccc}
F^{2} & -E F & z F^{2} & -z E F \\
-E F & E^{2} & -z E F & z E^{2} \\
z F^{2} & -z E F & z^{2} F^{2} & -z^{2} E F \\
-z E F & z E^{2} & -z^{2} E F & z^{2} E^{2}
\end{array}\right]
\end{aligned}
$$

where, $F=\left(r \sin \theta+y_{0}+\beta z\right), E=\left(r \cos \theta+x_{0}+\right.$ $\alpha z)$.

Its sequential principal minors are:

$$
\begin{aligned}
& \frac{F^{2}}{R^{3}} \geq 0 ; \frac{1}{R^{3}}\left|\begin{array}{cc}
F^{2} & -E F \\
-E F & E^{2}
\end{array}\right|=0 ; \\
& \frac{1}{R^{3}}\left|\begin{array}{ccc}
F^{2} & -E F & z F^{2} \\
-E F & E^{2} & -z E F \\
z F^{2} & -z E F & z^{2} F^{2}
\end{array}\right|=0 ; \\
& \frac{1}{R^{3}}\left|\begin{array}{cccc}
F^{2} & -E F & z F^{2} & -z E F \\
-E F & E^{2} & -z E F & z E^{2} \\
z F^{2} & -z E F & z^{2} F^{2} & -z^{2} E F \\
-z E F & z E^{2} & -z^{2} E F & z^{2} E^{2}
\end{array}\right|=0 .
\end{aligned}
$$

Therefore, $R(v ; u)$ is a convex function of $u$ and, as a result, $\bar{R}(u)$ is also convex.

To bring the discussion of the following problems more conveniently, we employ a set of maximal value points $\bar{I}$ and the associated mapping set $\bar{A}(u)$ as

$$
\begin{aligned}
\bar{I} & =\left\{i: i \in(1,2, \ldots, n) \text { and } R_{i}=\bar{R}\right\} \\
\bar{A}(u) & =\{\xi(i): i \in \bar{I}\} \\
\xi(i) & =\partial R_{i}(u)=\left(\frac{\partial R_{i}}{\partial x_{0}}, \frac{\partial R_{i}}{\partial y_{0}}, \frac{\partial R_{i}}{\partial \alpha}, \frac{\partial R_{i}}{\partial \beta}\right)^{T} \\
& {\left[\begin{array}{c}
\frac{r_{i} \cos \theta_{i}+x_{0}+\alpha z_{i}}{R_{i}} \\
\frac{r_{i} \sin \theta_{i}+y_{0}+\beta z_{i}}{R_{i}} \\
\frac{z_{i}\left(r_{i} \cos \theta_{i}+x_{0}+\alpha z_{i}\right)}{R_{i}} \\
\frac{z_{i}\left(r_{i} \sin \theta_{i}+y_{0}+\beta z_{i}\right)}{R_{i}}
\end{array}\right] }
\end{aligned}
$$

The minimum zone condition is closely related to the set of maximal value points $\bar{I}$. These points are valid constraints between the enclosure cylinder and the evaluated spatial line. Whether the value of $u$ satisfies the minimum condition depends only on the maximal value points and has nothing

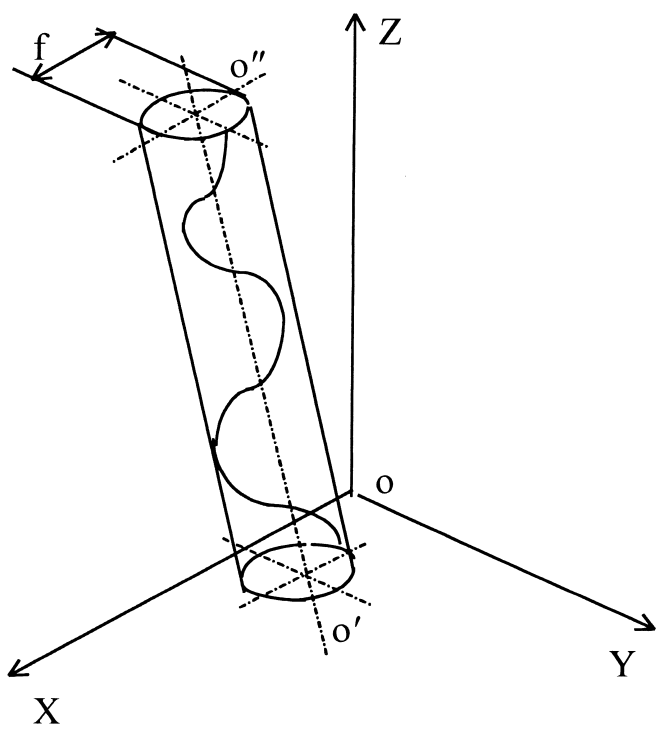

Fig. 2. The tilted cylinder enclosing a spatial line.

to do with the other points. Then, we seek the relationship between the maximal value $\bar{I}$ and the criterion of the minimum zone condition. The representative formula for the subgradient $\partial \bar{R}(u)$ can be derived using the use of the following lemma [9].

\subsection{Lemma 1}

Assuming $S$ is a nonempty convex set in $E^{n}, f_{1}, f_{2}: S \rightarrow$ $E^{1}$ are convex functions, then the subgradient set of function $\bar{f}=\max \left(f_{1}, f_{2}\right)$ can be denoted as follows:

$$
\partial \bar{f}=\partial\left(\max \left\{f_{1}, f_{2}\right\}\right)=\operatorname{conv}\left(\partial \overline{f_{1}} \cup \partial \overline{f_{2}}\right)
$$

where

$$
\partial \bar{f}_{i}=\left\{\begin{array}{c}
\partial f, f_{i}=\bar{f}=\max \left\{f_{1}, f_{2}\right\} \\
\phi, f_{i}<\bar{f}=\max \left\{f_{1}, f_{2}\right\}
\end{array}\right.
$$

$\operatorname{conv}\left(\partial \overline{f_{1}} \cup \partial \overline{f_{2}}\right)$ represents the convex hull of $\left(\partial \overline{f_{1}} \cup\right.$ $\left.\partial \overline{f_{2}}\right)$, and $\phi$ represents an empty set.

According to Lemma 1 , for $\bar{R}(u)=\max \left\{R_{1}, R_{2}, \ldots\right.$, $\left.R_{i}, \ldots R_{n}\right\}$, we can easily acquire

$$
\begin{aligned}
\partial \bar{R}(u) & =\partial\left(\max \left\{R_{1}, R_{2}, \ldots, R_{i}, \ldots, R_{n}\right\}\right) \\
& =\operatorname{conv}\left(\cup_{i \in \hat{I}} \partial \bar{R}_{i}\right)
\end{aligned}
$$

From Eq. (12) and Eq. (13), we have

$$
\underset{i \in \hat{I}}{\cup} \partial \bar{R}_{i}=[\xi(i): i \in \bar{I}]=\bar{A}(u),
$$

which yields

$$
\partial \bar{R}(u)=\operatorname{conv}[\bar{A}(u)]
$$

By its nature, the necessary and sufficient conditions for the convex function $f(x)$ to be a global minimum at a certain 


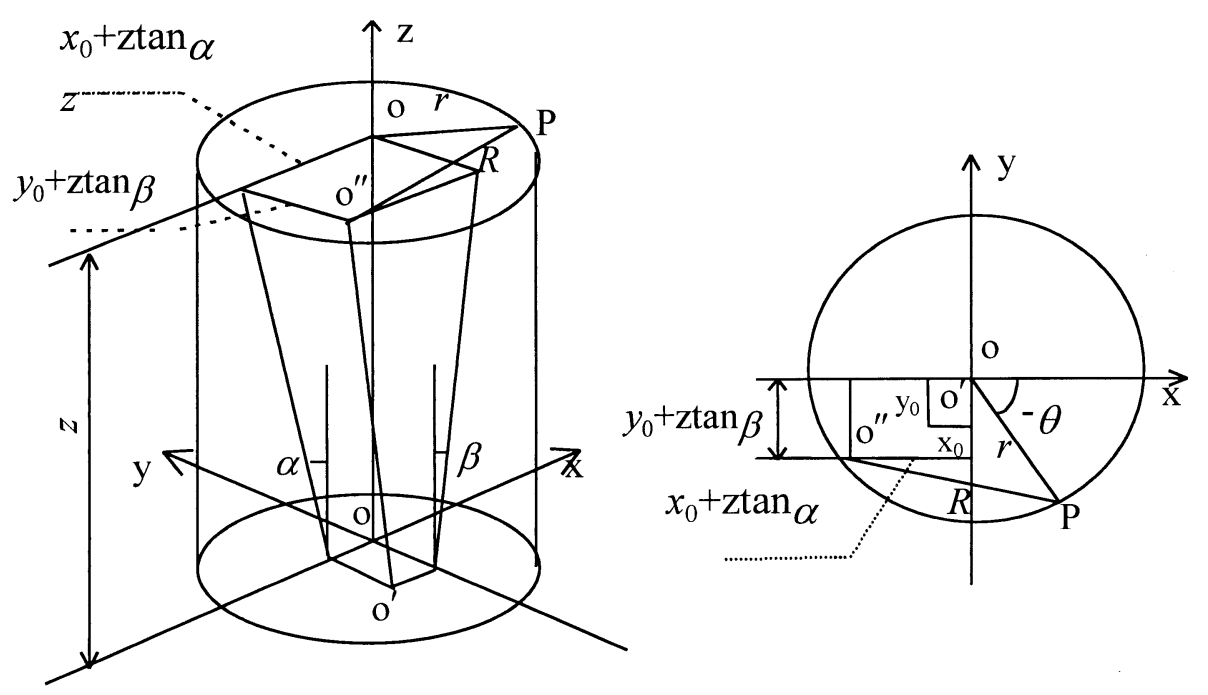

Fig. 3. The geometric location of the central axis of the tilted cylinder.

point in the open domain should be: $0 \in \partial f(x)$. So we can conclude that the necessary and sufficient condition of minimizing $\bar{R}(u)$ is: $0 \in \partial \vec{R}(u)$. According to Eq. (14), we have

$$
0 \in \operatorname{conv}[\bar{A}(u)]
$$

Eq. (15) gives the criterion for the minimum zone condition. According to this criterion, we then draw the conclusion that only when the convex hull of $\bar{A}(u)$ encloses the origin, can the alignment variable, $u=\left(x_{0}, y_{0}, \alpha, \beta\right)^{T}$, make $\bar{R}(u)$ minimal.

\section{Computational method}

The convex hull of the mapping set $\bar{A}(u)$ of maximal value points is a problem in a four-dimensional (4-D) space. In the analysis of spatial straightness errors, it is difficult to represent the problem in a three-dimensional (3-D) space geometrically. Therefore, an algebraic judgment formula based on Eq. (15) can be constructed for the computer computation according to the definition of a convex hull: [10]

$$
\left\{\begin{array}{l}
\sum_{i=1}^{h} \lambda_{i} \xi(i)=0 \\
\sum_{i=1}^{h} \lambda_{i}=1 \\
\lambda_{i} \geq 0 \\
i=1,2, \ldots, h
\end{array}\right.
$$

Eqs. (16) provide a minimum zone judgment condition for the outer enclosure cylinder, where $h$ is the number of the maximal value point set $\bar{I}$. Whether the outer enclosure cylinder of the spatial line fits the criterion of the minimum zone condition depends only on the set of maximal value points. For a cylinder, because its maximal value points are valid constraints, the number of these points must be equivalent to the number of unknowns in the mathematical model. Because there are five unknown coefficients in Eq. (2) - $R, x_{0}, y_{0}, \alpha$, and $\beta$ - the number of maximal value points should normally be five. In Eqs. (16), the first two equations are linear equations from which $\lambda_{i}$ can be calculated. The following inequality, $\lambda_{i} \geq 0$, can be used as the minimum judgment condition. When the minimum zone condition is satisfied, $\lambda_{i}$ will be in either one of the following two cases.

First, in the normal case, all $\lambda_{i}$ are greater than zero. Because no $\lambda_{i}$ equals zero, the formula

$$
\sum_{i=1}^{h} \lambda_{i} \xi(i)=0
$$

includes the influence of all $\xi(i)$; that is, the maximal value points determined by $\xi(i)$ are valid constraints. Hence, the number of the maximal value points is $h=5$.

Second, in the abnormal case, it should also be noticed that the degenerated phenomenon may sometimes occur. At this state, all $\lambda_{i}$ are non-negative, but certain $\lambda_{i}$ may be equal to zero. For example when $\lambda_{k}=0$, where $k \in \bar{I}$, the item $\lambda_{k} \xi(k)$ equals zero, so that the minimum judgment condition will not include the influence of $\xi(k)$. Therefore, the point decided by $\xi(k)$ will not be a valid constraint and can be removed. The number of contour points will decrease; that is, $h<5$. Regardless the value of $h$, it is concluded that the minimum zone condition still can be satisfied as long as Eqs. (16) are satisfied.

In this study, the computer judgment scheme proceeds with the following steps. 
Step 1. Input the measured points in $(r, \theta, z)$ and the number of measured points $n$. Let $u=0$.

Step 2. Sequentially select five original points $i_{1}, i_{2}, i_{3}$, $i_{4}, i_{5}$ as equidistant points with respect to the $o^{\prime} o^{\prime \prime}$ axis, and extract the value of $u$ by solving (4-D) nonlinear equations.

Step 3. Extract the values of maximal value points and the set of maximal value points.

Step 4. Judge whether the minimum condition is satisfied by the use of Eqs. (16). If yes, print out results and stop. If not, go to Step 5.

Step 5. Select a new point to substitute one of the five original points and return to Step 2.

In Step 2, we extract the values of the alignment variables $u$ by solving (4-D) nonlinear equations, and assume that the five original points selected sequentially are points that are equidistant with respect to the $o^{\prime} o^{\prime \prime}$ axis; that is, $i=1,2,3,4,5$. The difference between any two distances from any two points $i_{j} s$ to the $o^{\prime} o^{\prime \prime}$ axis, respectively, should be zero. We then solve the following system equations.

$$
\begin{aligned}
P\left(x_{0}, y_{0}, \alpha, \beta\right)= & P\left(x_{0 i}, y_{0 i}, \alpha_{i}, \beta_{i}\right)+\left.a \frac{\partial P}{\partial x_{0}}\right|_{u_{i}} \\
& +\left.b \frac{\partial P}{\partial y_{0}}\right|_{u_{i}} \\
& +\left.c \frac{\partial P}{\partial \alpha}\right|_{u_{i}}+\left.d \frac{\partial P}{\partial \beta}\right|_{u_{i}}+\cdots \\
Q\left(x_{0}, y_{0}, \alpha, \beta\right)= & Q\left(x_{0 i}, y_{0 i}, \alpha_{i}, \beta_{i}\right)+\left.a \frac{\partial Q}{\partial x_{0}}\right|_{u_{i}} \\
& +\left.b \frac{\partial Q}{\partial y_{0}}\right|_{u_{i}}+\left.c \frac{\partial Q}{\partial \alpha}\right|_{u_{i}} \\
& +\left.d \frac{\partial Q}{\partial \beta}\right|_{u_{i}}+\cdots
\end{aligned}
$$

$$
\begin{aligned}
\{M(u)= & {\left[\left(r_{1} \cos \theta_{1}+x_{0}+\alpha z_{1}\right)^{2}+\left(r_{1} \sin \theta_{1}+y_{0}+\beta z_{1}\right)^{2}\right] } \\
& -\left[\left(r_{2} \cos \theta_{2}+x_{0}+\alpha z_{2}\right)^{2}+\left(r_{2} \sin \theta_{2}+y_{0}+\beta z_{2}\right)^{2}\right]=0 \\
N(u)= & {\left[\left(r_{1} \cos \theta_{1}+x_{0}+\alpha z_{1}\right)^{2}+\left(r_{1} \sin \theta_{1}+y_{0}+\beta z_{1}\right)^{2}\right] } \\
& -\left[\left(r_{3} \cos \theta_{3}+x_{0}+\alpha z_{3}\right)^{2}+\left(r_{3} \sin \theta_{3}+y_{0}+\beta z_{3}\right)^{2}\right]=0 \\
P(u)= & {\left[\left(r_{1} \cos \theta_{1}+x_{0}+\alpha z_{1}\right)^{2}+\left(r_{1} \sin \theta_{1}+y_{0}+\beta z_{1}\right)^{2}\right] } \\
& -\left[\left(r_{4} \cos \theta_{4}+x_{0}+\alpha z_{4}\right)^{2}+\left(r_{4} \sin \theta_{4}+y_{0}+\beta z_{4}\right)^{2}\right]=0 \\
Q(u)= & {\left[\left(r_{1} \cos \theta_{1}+x_{0}+\alpha z_{1}\right)^{2}+\left(r_{1} \sin \theta_{1}+y_{0}+\beta z_{1}\right)^{2}\right] } \\
& -\left[\left(r_{5} \cos \theta_{5}+x_{0}+\alpha z_{5}\right)^{2}+\left(r_{5} \sin \theta_{5}+y_{0}+\beta z_{5}\right)^{2}\right]=0
\end{aligned}
$$

The iterative method is adopted when solving systems of nonlinear Eqs. (17). $u\left(x_{0}, y_{0}, \alpha, \beta\right)=0$ is chosen as the original value. Assuming $u_{r}=\left(x_{0 r}, y_{0 r}, \alpha_{r}, \beta_{r}\right)$ is the exact solution of the equations, and $u_{i}=\left(x_{0 i}, y_{0 i}, \alpha_{i}, \beta_{i}\right)$ is an approximate solution. $M(u), N(u), P(u), Q(u)$ can be expanded by the Taylor series at $u_{i}$.

$$
\begin{aligned}
M\left(x_{0}, y_{0}, \alpha, \beta\right)= & M\left(x_{0 i}, y_{0 i}, \alpha_{i}, \beta_{i}\right)+\left.a \frac{\partial M}{\partial x_{0}}\right|_{u_{i}} \\
& +\left.b \frac{\partial M}{\partial y_{0}}\right|_{u_{i}}+\left.c \frac{\partial M}{\partial \alpha}\right|_{u_{i}}+\left.d \frac{\partial M}{\partial \beta}\right|_{u_{i}}+\cdots \\
N\left(x_{0}, y_{0}, \alpha, \beta\right)= & N\left(x_{0 i}, y_{0 i}, \alpha_{i}, \beta_{i}\right)+\left.a \frac{\partial N}{\partial x_{0}}\right|_{u_{i}} \\
& +\left.b \frac{\partial N}{\partial y_{0}}\right|_{u_{i}}+\left.c \frac{\partial N}{\partial \alpha}\right|_{u_{i}}+\left.d \frac{\partial N}{\partial \beta}\right|_{u_{i}}+\ldots
\end{aligned}
$$

where

$$
\left\{\begin{array}{c}
x_{0 r}=x_{0 i}+a \\
y_{0 r}=y_{0 i}+b \\
\alpha_{r}=\alpha_{i}+c \\
\beta_{r}=\beta_{i}+d
\end{array}\right.
$$

We kept only the first-order differential terms of the Taylor series in Eq. (18) and truncated the higher-order terms, then the linear equations with four unknown coefficients $a, b, c, d$ were obtained. However, because the truncation of higher-order terms, $a, b, c, d$ correspond to the approximate values of $\left(x_{0 r}-x_{0 i}\right),\left(y_{0 r}-y_{0 i}\right),\left(\alpha_{r}-\right.$ $\left.\alpha_{i}\right),\left(\beta_{r}-\beta_{i}\right)$, respectively. Therefore, even if the values of $a, b, c, d$ are extracted, we can only get an approximate solution for $x_{0}, y_{0}, \alpha, \beta$; that is 
Table 1

Measured data of example 1

\begin{tabular}{|c|c|c|c|c|c|c|c|c|c|c|c|c|}
\hline Measured points & 1 & 2 & 3 & 4 & 5 & 6 & 7 & 8 & 9 & 10 & 11 & 12 \\
\hline$Z(\mathrm{~cm})$ & 5 & 10 & 15 & 20 & 25 & 30 & 35 & 40 & 45 & 50 & 55 & 60 \\
\hline$r(\mu \mathrm{m})$ & 6.1 & 12.6 & 11.2 & 15.0 & 4.2 & 12.0 & 9.2 & 10.5 & 16.0 & 11.0 & 8.4 & 3.7 \\
\hline$\theta\left(^{0}\right)$ & 34 & 190.5 & 159 & 232 & 70 & 8 & 176 & 234 & 342 & 263 & 46 & 12 \\
\hline
\end{tabular}

$$
\left\{\begin{aligned}
x_{0 i+1} & =x_{0 i}+a \\
y_{0 i+1} & =y_{0 i}+b \\
\alpha_{i+1} & =\alpha_{i}+c \\
\beta_{i+1} & =\beta_{i}+d
\end{aligned}\right.
$$

where, $x_{0 i+1}, y_{0 i+1}, \alpha_{i+1}, \beta_{i+1}$ are the more accurate approximate values of $x_{0}, y_{0}, \alpha, \beta$. Because Eq. (16) is the iterative formula, we can extract more accurate values for $x_{0}, y_{0}, \alpha, \beta$ by substituting $x_{0 i+1}, y_{0 i+1}, \alpha_{i+1}, \beta_{i+1}$ into Eq. (19). The iterative process ends, until the values of $x_{0}$, $y_{0}, \alpha, \beta$ are stable; that is, the difference of the values obtained in the two successive steps is very small. Iterative results from the software developed by the authors are very steady and show that the deviation of the iteration can be converged to zero.

Substituting $u$ obtained from the above calculation into Eq. (2), we can get an $R$ value for all measured points. After comparison, the maximal value $\bar{R}(u)$, and the set of maximal value points $\bar{I}$ can be extracted.

\section{Practical examples}

\subsection{Example 1}

Table 1 shows the measured data of the spatial straightness error of a linear stage. The final results of the error evaluation are shown in Table 3. Because all $\lambda_{i}$ are greater than zero, the evaluated results satisfy the minimum condi-

Table 2

Midcalculation results of example 1

Maximal value points: $\bar{I}=\{1,3,4,6,9\}$

$x_{0}=4.536636, y_{0}=4.897267, \alpha=-0.000019, \beta=0.000006$

$\bar{R}=11.7791$

$\lambda_{1}=-0.076231, \lambda_{2}=0.369570, \lambda_{3}=0.467130, \lambda_{4}=0.073406$,

$\lambda_{5}=0.166125$

$f=2 \bar{R}=23.5582$

Table 3

Final evaluated results of example 1

Maximal value points: $\bar{I}=\{1,3,4,6,7\}$

$x_{0}=4.640799, y_{0}=4.743749, \alpha=-0.000019$,

$\beta=-0.000006$

$\bar{R}=11.763077$

$\lambda_{1}=0.090856, \lambda_{2}=0.315581, \lambda_{3}=0.403747, \lambda_{4}=0.035690$,

$\lambda_{5}=0.154126$

$f=2 \bar{R}=23.5262$ tion. The evaluated spatial straightness error is $23.5262 \mu \mathrm{m}$. In the results shown in Table $2, \lambda_{1}=-0.076231<0$ appears, so the minimum condition has not been satisfied. Table 4 gives the evaluated results using the least-squares method. The minimal enclosure cylinder of the measured data is shown in Fig. 4.

\subsection{Example 2}

The measured data of a table moving along a machine tool guide way is shown in Table 5. The final results of the error evaluation are shown in Table 7. The spatial straightness error of the guide way is $32.57 \mu \mathrm{m}$. The intermittent results shown in Table 6 have $\lambda_{5}=-0.03509$, so the minimum condition has not been satisfied. Table 8 gives the evaluated results using the least-squares method.

Table 4

Evaluated results by LSQ of example 1

Coefficient of least-squares line: $a=-7.0764 ; b=-0.0776$;
$\quad c=-0.0437 ; d=0.2031$
$f=26.9414$

$f=26.9414$

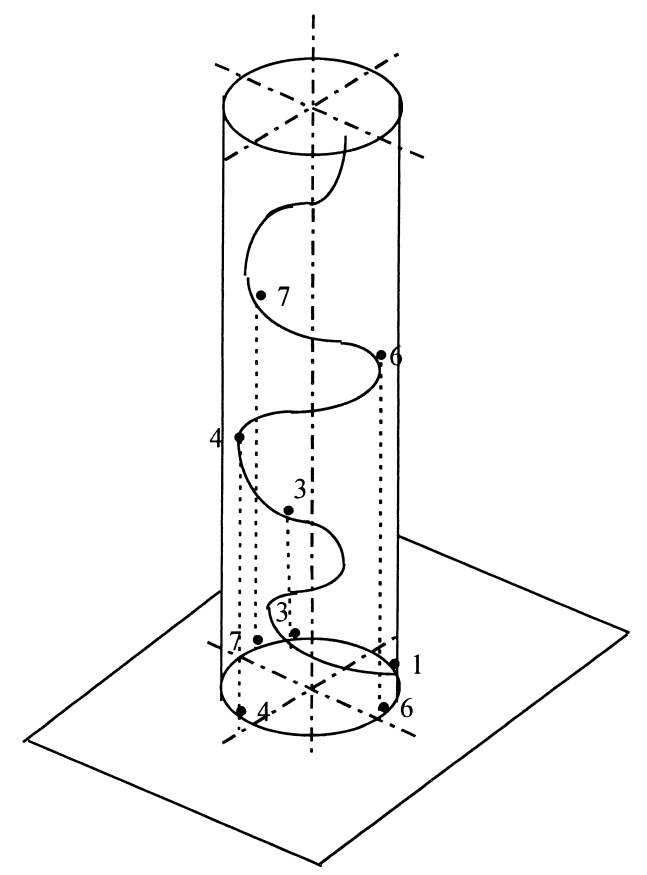

Fig. 4. The minimal cylinder enclosure the measured data. 
Table 5

Measured data of example 2

\begin{tabular}{|c|c|c|c|c|c|c|c|c|c|c|c|c|}
\hline Measured points & 1 & 2 & 3 & 4 & 5 & 6 & 7 & 8 & 9 & 10 & 11 & 12 \\
\hline$Z(\mathrm{~cm})$ & 5 & 10 & 15 & 20 & 25 & 30 & 35 & 40 & 45 & 50 & 55 & 60 \\
\hline$r(\mu \mathrm{m})$ & 13.2 & 9.8 & 16.9 & 18.3 & 17.0 & 15.2 & 10.3 & 9.0 & 12.4 & 16.8 & 11.0 & 6.3 \\
\hline$\theta\left({ }^{0}\right)$ & 12 & 78 & 124 & 46 & 178 & 256 & 224 & 289 & 310 & 345 & 45 & 16 \\
\hline
\end{tabular}

\section{Geometric judgment method}

Eq. (15), the criterion for the minimum zone condition, has an apparent geometrical meaning; that is, the convex hull of the mapping set $\bar{A}(u)$ of maximal value points encloses the origin. The dimension of $\xi(i)$ indicates the mapping space; that is, the mapping coordinate system. From Eq. (13), we know $\xi(i)$ is the coordinate matrix for every measured point in the mapping coordinate system.

$$
\xi(i)=\left(\tau_{1 i}, \tau_{2 i}, \tau_{3 i}, \tau_{4 i}\right)^{T}=\left(\frac{\partial R_{i}}{\partial x_{0}}, \frac{\partial R_{i}}{\partial y_{0}}, \frac{\partial R_{i}}{\partial \alpha}, \frac{\partial R_{i}}{\partial \beta}\right)^{T}
$$

where

$$
\begin{aligned}
\tau_{1 i} & =\frac{\partial R_{i}}{\partial x_{0}}=\frac{r_{i} \cos \theta_{i}+x_{0}+\alpha z_{i}}{R_{i}} \\
\tau_{2 i} & =\frac{\partial R_{i}}{\partial y_{0}}=\frac{r_{i} \sin \theta_{i}+y_{0}+\beta z_{i}}{R_{i}} \\
\tau_{3 i} & =\frac{\partial R_{i}}{\partial \alpha}=\frac{z_{i}\left(r_{i} \cos \theta_{i}+x_{0}+\alpha z_{i}\right)}{R_{i}} \\
\tau_{4 i} & =\frac{\partial R_{i}}{\partial \beta}=\frac{z_{i}\left(r_{i} \sin \theta_{i}+y_{0}+\beta z_{i}\right)}{R_{i}}
\end{aligned}
$$

Table 6

Midcalculation results of example 2

Maximal value points: $\bar{I}=\{1,4,5,6,11\}$

$x_{0}=2.212072, y_{0}=-11.107992, \alpha=-0.000005$,

$\beta=0.000033$

$\bar{R}=16.301811$

$\lambda_{1}=0.096840, \lambda_{2}=0.383047, \lambda_{3}=0.285638, \lambda_{4}=0.285638$,

$\lambda_{5}=-0.035090$

$f=2 \bar{R}=32.6036$

Table 7

Final evaluated results of example 2

Maximal value points: $\bar{I}=\{4,5,6,10,11\}$

$x_{0}=1.6496, y_{0}=-10.6815, \alpha=-0.000003, \beta=0.000031$

$\bar{R}=16.285259$

$\lambda_{1}=0.0252, \lambda_{2}=0.219, \lambda_{3}=0.324, \lambda_{4}=0.356, \lambda_{5}=0.076$

$f=2 \bar{R}=32.5705$

Table 8

Evaluated results of example 2 by LSQ method

Coefficient of least-squares line: $a=0.3075 ; b=6.9422$;

$c=-0.1988 ; d=0.0396$

$f=36.6764$
Having substituted the obtained $u$, that is, $\left(x_{0}, y_{0}, \alpha, \beta\right)$ and the cylindrical coordinates $(r, \theta, z)$ of the maximal value points into Eq. (20), we can attain the value of $\xi(i)$ of every point; that is, the projecting points of the mapping space. The figure enclosed by linking lines between the projecting points is just the convex hull of $\bar{A}(u)$. Because $\xi(i)$ is a (4-D) variable, $\bar{A}(u)$ are mapped in (4-D) space, then a convex hull in (4-D) space can be acquired. If the convex hull encloses the origin, the criterion of the minimum zone condition will be satisfied. However, a convex hull in (4-D) space is difficult to represent graphically, so we should project it onto each coordinate plane (2-D) of the (4-D) space, and draw the projected convex set in each coordinate plane. The judgment of the minimum zone condition will be carried out on each of the planar projected figures.

We assume that the (4-D) orthogonal coordinates system is $\left(\tau_{1}, \tau_{2}, \tau_{3}, \tau_{4}\right)$, which consists of six coordinate planes; namely, $\left(\tau_{1}, \tau_{2}\right),\left(\tau_{1}, \tau_{3}\right),\left(\tau_{1}, \tau_{4}\right),\left(\tau_{2}, \tau_{3}\right),\left(\tau_{2}, \tau_{4}\right)$ and $\left(\tau_{3}, \tau_{4}\right)$ planes. According to the theory of convex analysis [9], we have the following geometric judgment condition: only when all the projected convex sets for the six coordinate planes enclose the origin, can the minimum zone condition be satisfied.

The geometrical judgment of the spatial straightness error evaluation given in Example 1 above is carried out as follows. Mapping of the five contour points in (4-D) space occurs as follows:

$$
\begin{aligned}
\xi(1) & =\left(\tau_{11}, \tau_{21}, \tau_{31}, \tau_{41}\right)^{T} \\
& =(0.744,0.668,37181,33400)^{T} ; \\
\xi(2) & =\left(\tau_{12}, \tau_{22}, \tau_{32}, \tau_{42}\right)^{T} \\
& =(-0.737,0.688,-110509,100200)^{T} ; \\
\xi(3) & =\left(\tau_{13}, \tau_{23}, \tau_{33}, \tau_{43}\right)^{T} \\
& =(-0.714,-0.704,-142800,-140800)^{T} ; \\
\xi(4) & =\left(\tau_{14}, \tau_{24}, \tau_{34}, \tau_{44}\right)^{T} \\
& =(0.920,0.392,276000,117600)^{T} ; \\
\xi(5) & =\left(\tau_{15}, \tau_{25}, \tau_{35}, \tau_{45}\right)^{T} \\
& =(-0.951,0.279,-332850,976501)^{T} .
\end{aligned}
$$

Geometric judgment graphs are shown in Fig. 5.

It is clear, from Fig. 5, that all of the six projected diagrams enclose the origin, which means that the minimum 

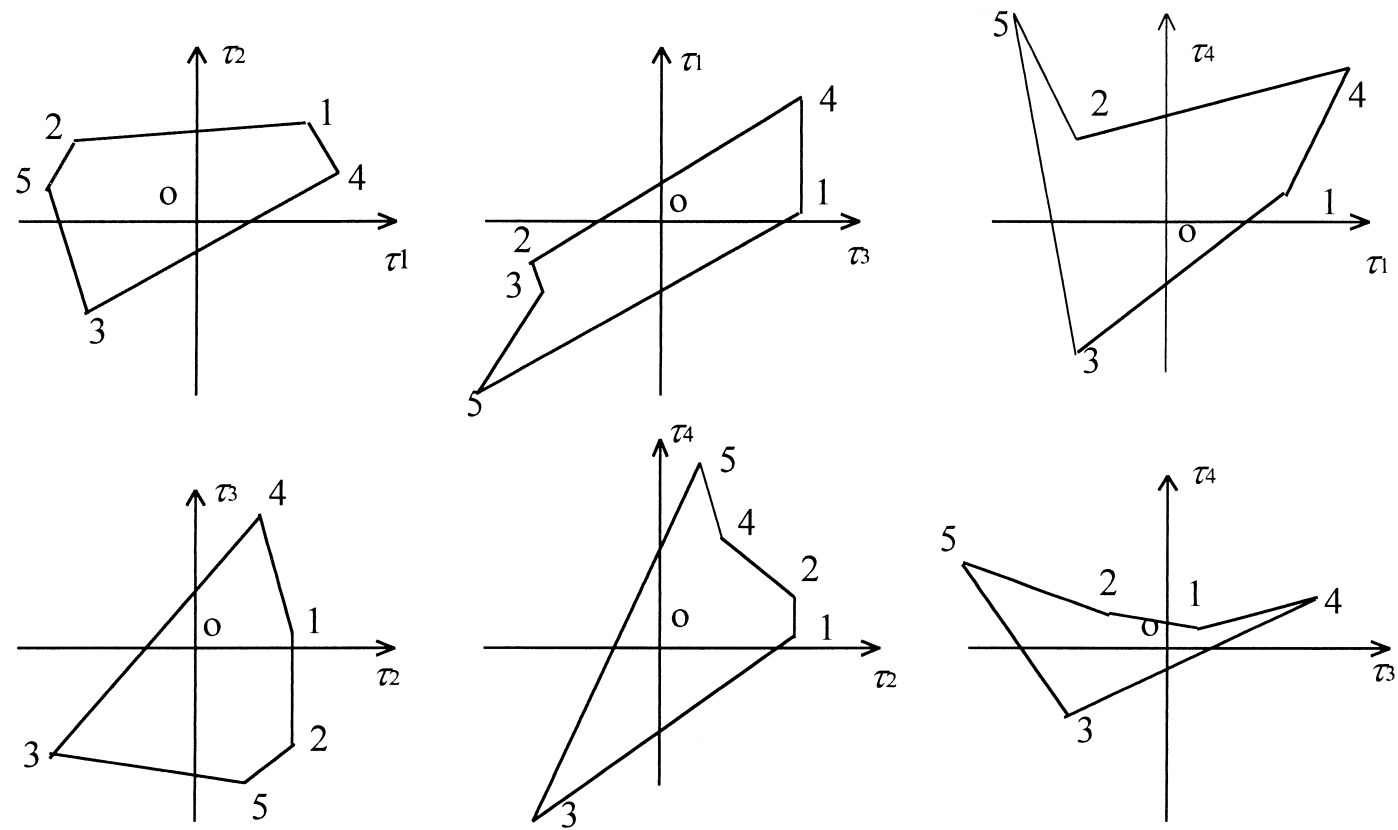

Fig. 5. Geometrical judgment graphs of the minimum zone condition.

zone condition has been satisfied. This conclusion is the same as that of the algebraic judgment. The two methods can be used to verify one another.

\section{Conclusions}

A new and comprehensive method for the spatial straightness error evaluation was proposed based on the minimum zone condition in this paper. The criterion for the minimum zone condition was proposed to ensure the existence and uniqueness of the solution. A computational algorithm was implemented to obtain the results rapidly. The correctness of the method can be demonstrated by use of geometric judgment method. The proposed method is very practical in use and provides an ideal means to evaluate the spatial straightness error using a personal computer.

\section{References}

[1] ISO R1101. Technical drawings-Geometrical tolerancing. Geneva: International Organization for Standardization, 1983.

[2] Chetwynd DG. Applications of linear programming to engineering metrology. Proc. Inst. Mech Eng. 1985;199:93-100.

[3] Fukuda M, Shimokohbe A. Algorithms for form evaluation methods for minimum zone and least squares. Proc. Int. Symp. Metrology for Quality Production, Tokyo, 1984, pp. 197-202.

[4] Shunmugam MS. Comparison of linear and normal deviations of forms of engineering surfaces. Prec. Eng. 1987;9:96-102.

[5] Traband MT, Joshi S, Wysk RA, Cavalier TM. Evaluation of straightness and flatness tolerances using minimum zone. Manufact. Rev. 1989;2:189-95.

[6] Carr K, Ferreira P. Verification of form tolerances, Part II: Cylindricity and straightness of a median line. Prec. Eng. 1995;17:144-156.

[7] Suen DS, Chang CN. Application of neural network interval regression method for minimum zone straightness and flatness. Prec. Eng. 1997;20:196-207.

[8] Huang ST, Fan KC, Wu, John H. A new minimum zone method for evaluating straightness errors. Prec. Eng. 1993;15:158-165.

[9] Rockerfeller RT. Convex Analysis. Princeton, NJ: Princeton University Press, 1970

[10] Yu YS. Principle and Method of Mathematical Programming. Wuhan, China, HUST Press, 1993. 